\section{A Wandering Mind Is an Unhappy Mind}

Matthew A. Killingsworth* and Daniel T. Gilbert

U nlike other animals, human beings spend a lot of time thinking about what is not going on around them, contemplating events that happened in the past, might happen in the future, or will never happen at all. Indeed, "stimulus-independent thought" or "mind wandering" appears to be the brain's default mode of operation (1-3). Although this ability is a remarkable evolutionary achievement that allows people to learn, reason, and plan, it may have an emotional cost. Many philosophical and religious traditions teach that happiness is to be found by living in the moment, and practitioners are trained to resist mind wandering and "to be here now." These traditions suggest that a wandering mind is an unhappy mind. Are they right?

Laboratory experiments have revealed a great deal about the cognitive and neural bases of mind wandering (3-7), but little about its emotional consequences in everyday life. The most reliable method for investigating real-world emotion is experience sampling, which involves contacting people as they engage in their everyday activities and asking them to report their thoughts, feelings, and actions at that moment. Unfortunately, collecting real-time reports from large numbers of people as they go about their daily lives is so cumbersome and expensive that experience sampling has rarely been used to investigate the relationship between mind wandering and happiness and has always been limited to very small samples $(8,9)$.

We solved this problem by developing a Web application for the iPhone (Apple Incorporated, Cupertino, California), which we used to create an unusually large database of real-time reports of thoughts, feelings, and actions of a broad range of people as they went about their daily activities. The application contacts participants through their iPhones at random moments during their waking hours, presents them with questions, and records their answers to a database at www. trackyourhappiness.org. The database currently contains nearly a quarter of a million samples from about 5000 people from 83 different countries who range in age from 18 to 88 and who collectively represent every one of 86 major occupational categories.

To find out how often people's minds wander, what topics they wander to, and how those wanderings affect their happiness, we analyzed samples from 2250 adults $(58.8 \%$ male, $73.9 \%$ residing in the United States, mean age of 34 years) who were randomly assigned to answer a happiness question ('How are you feeling right now?') answered on a continuous sliding scale from very bad (0) to very good (100), an activity question ("What are you doing right now?") answered by endorsing one or more of 22 activities adapted from the day reconstruction method $(10,11)$, and a mind-wandering question ("Are you thinking about something other than what you're currently doing?') answered with one of four options: no; yes, something pleasant; yes, something neutral; or yes, something unpleasant. Our analyses revealed three facts.

First, people's minds wandered frequently, regardless of what they were doing. Mind wandering occurred in $46.9 \%$ of the samples and in at least $30 \%$ of the samples taken during every activity except making love. The frequency of mind wandering in our real-world sample was considerably higher than is typically seen in laboratory experiments. Surprisingly, the nature of people's activities had only a modest impact on whether their minds wandered and had almost no impact on the pleasantness of the topics to which their minds wandered (12).

Second, multilevel regression revealed that people were less happy when their minds were wandering than when they were not [slope $(b)=-8.79$, $P<0.001]$, and this was true during all activities,

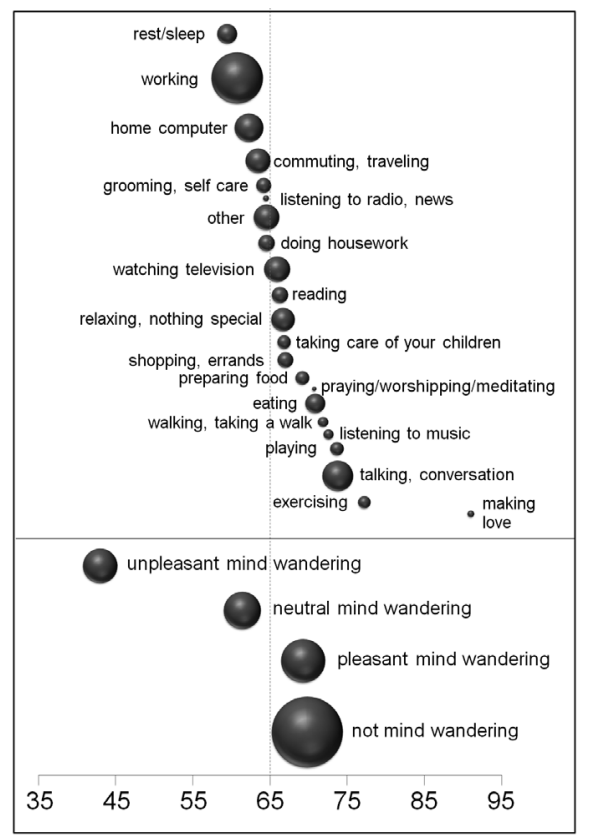

Fig. 1. Mean happiness reported during each activity (top) and while mind wandering to unpleasant topics, neutral topics, pleasant topics or not mind wandering (bottom). Dashed line indicates mean of happiness across all samples. Bubble area indicates the frequency of occurrence. The largest bubble ("not mind wandering") corresponds to $53.1 \%$ of the samples, and the smallest bubble ("praying/worshipping/meditating") corresponds to $0.1 \%$ of the samples. including the least enjoyable. Although people's minds were more likely to wander to pleasant topics ( $42.5 \%$ of samples) than to unpleasant topics ( $26.5 \%$ of samples) or neutral topics ( $31 \%$ of samples), people were no happier when thinking about pleasant topics than about their current activity $(b=$ -0.52 , not significant) and were considerably unhappier when thinking about neutral topics $(b=$ $-7.2, P<0.001)$ or unpleasant topics $(b=-23.9$, $P<0.001$ ) than about their current activity (Fig. 1, bottom). Although negative moods are known to cause mind wandering (13), time-lag analyses strongly suggested that mind wandering in our sample was generally the cause, and not merely the consequence, of unhappiness (12).

Third, what people were thinking was a better predictor of their happiness than was what they were doing. The nature of people's activities explained $4.6 \%$ of the within-person variance in happiness and $3.2 \%$ of the between-person variance in happiness, but mind wandering explained $10.8 \%$ of within-person variance in happiness and $17.7 \%$ of between-person variance in happiness. The variance explained by mind wandering was largely independent of the variance explained by the nature of activities, suggesting that the two were independent influences on happiness.

In conclusion, a human mind is a wandering mind, and a wandering mind is an unhappy mind. The ability to think about what is not happening is a cognitive achievement that comes at an emotional cost.

References and Notes

1. M. E. Raichle et al., Proc. Natl. Acad. Sci. U.S.A. 98, 676 (2001)

2. K. Christoff, A. M. Gordon, ]. Smallwood, R. Smith, J. W. Schooler, Proc. Natl. Acad. Sci. U.S.A. 106, 8719 (2009).

3. R. L. Buckner, ]. R. Andrews-Hanna, D. L. Schacter, Ann. N. Y. Acad. Sci. 1124, 1 (2008).

4. J. Smallwood, J. W. Schooler, Psychol. Bull. 132, 946 (2006).

5. M. F. Mason et al., Science 315, 393 (2007).

6. J. Smallwood, E. Beach, J. W. Schooler, T. C. Handy, J. Cogn. Neurosci. 20, 458 (2008).

7. R. L. Buckner, D. C. Carroll, Trends Cogn. Sci. 11, 49 (2007)

8. J. C. McVay, M. J. Kane, T. R. Kwapil, Psychon. Bull. Rev. 16, 857 (2009)

9. M. J. Kane et al., Psychol. Sci. 18, 614 (2007).

10. D. Kahneman, A. B. Krueger, D. A. Schkade, N. Schwarz, A. A. Stone, Science 306, 1776 (2004).

11. A. B. Krueger, D. A. Schkade, J. Public Econ. 92, 1833 (2008).

12. Materials and methods are available as supporting material on Science Online.

13. J. Smallwood, A. Fitzgerald, L. K. Miles, L. H. Phillips, Emotion 9, 271 (2009).

14. We thank V. Pitiyanuvath for engineering www. trackyourhappiness.org and R. Hackman, A. Jenkins, W. Mendes, A. Oswald, and T. Wilson for helpful comments.

Supporting Online Material

www.sciencemag.org/cgi/content/full/330/6006/932/DC1 Materials and Methods

Table $\mathbf{S 1}$

References

18 May 2010; accepted 29 September 2010 10.1126/science.1192439

Harvard University, Cambridge, MA 02138, USA.

*To whom correspondence should be addressed. E-mail: mkilling@fas.harvard.edu 


\section{Science \\ nIAAAS}

\section{Supporting Online Material for}

\section{A Wandering Mind Is an Unhappy Mind}

Matthew A. Killingsworth and Daniel T. Gilbert*

*To whom correspondence should be addressed. E-mail: gilbert@wjh.harvard.edu

Published 12 November 2010, Science 330, 932 (2010)

DOI: $10.1126 /$ science.1192439

\section{This PDF file includes:}

Materials and Methods

Table S1

References 


\section{Supporting Online Material}

\section{Participants and Procedure}

1. We stated "The most reliable method for investigating real-world emotion is experience sampling.” Experience sampling is generally considered the "gold standard” for investigating real-time emotion in vivo because it reduces or eliminates many of the potential biases inherent in other survey methodologies (S1). For example, surveys normally require people to report on emotional experiences long after they are over (“How did you feel last week?”) and to integrate experiences over time ("In general, how happy have you been in the last year?”). Although answers to such questions are good predictors of many consequential outcomes, they are poor indices of people's momentary emotional experiences because both memory and integration are notoriously susceptible to error $(S 2, S 3)$. In addition, surveys normally ask people to report on many aspects of their lives (e.g., income, health, etc.), and answering such questions can distort subsequent reports of emotional experiences (S4).

2. Participants volunteered for the study by signing up at trackyourhappiness.org. Our only advertisement consisted of a link from our laboratory’s website to trackyourhappiness.org, though the project did receive significant national press coverage. At initial signup, participants completed an informed consent form in which they certified that they were at least 18 years old. Participants then answered several questions about themselves, one of which asked them to select their birth year from a drop-down menu. Twenty-seven of the 2,250 participants in our sample selected a birth year indicating they were less than 18 years old. Because these participants had already certified that they were at least 18 years old, and because selecting a birth year required a more complicated response than did certifying their age, we considered these to be response errors and included these participants in the data set. 
However, we omitted these participants’ ages when computing the mean age of the participants.

3. Next, participants were asked to indicate the times at which they typically woke up and went to sleep, and how many times during the day they wished to receive a sample request (default $=3$, minimum $=1$ ). A computer algorithm then divided each participant's day into a number of intervals equal to the number of samples to be requested, and a random time was chosen within each interval. New random times were generated each day, and the times were independently randomized for each participant. At each of these times, participants received a notification on their iPhone, asking them to respond to a variety of questions about their feelings, thoughts, behavior, and environment. Samples were collected on all days of the week.

4. Participants received requests for samples until they chose to discontinue participation. If 50 samples had been collected, sampling stopped for 6 months or until the participant requested that it be restarted.

5. In each sample, different questions had different probabilities of being asked. The happiness question and the activity question were asked in all samples, but the mind-wandering question was asked in a randomly selected subset of samples. Only those samples that were randomly assigned to include the mind-wandering question are reported here. The happiness question was always asked before the mind-wandering question, and when the mindwandering question was asked it was always asked before the activity question. Other questions were also asked but are not relevant to the present report. Participants contributed an average of 7.9 samples $(\mathrm{SD}=5.8$, range $=1$ to 39$)$ to the present report. 
6. We computed "compliance rate” by dividing the number of samples received by the number of samples requested during a participant's "active period,” which we defined as the interval between a participant's first and last response. For example, if a participant completed 40 samples at the time of analysis but the 40th sample corresponded to the 50th request, then that participant's compliance rate would be $80 \%$. Our mean compliance rate was $83 \%$ and our median compliance rate was 93\%. Calculation of compliance rates was based on all samples, and not only those in which the mind-wandering question was asked.

7. The list of activities was adapted from the Day Reconstruction Method (S1, S5). Participants appeared able to use this scheme to categorize their activities, as in only $6.7 \%$ of all samples did participants indicate “other” as their primary activity.

\section{Data Analyses}

8. Due to the nested structure of the data, analyses of sample-level data (with the exception of within-person variance calculations as detailed below) were performed using multilevel regression with samples nested within persons. These analyses were performed in R using the function lmer from the lme4 package. Between-person analyses did not contain nested data and were performed using OLS regression.

9. We stated that "the nature of people's activities had only a modest impact on whether their minds wandered.” Evidence for this statement includes the facts that (a) there was a consistently high rate of mind-wandering across all activities except for making love, and (b) the nature of people's activities explained only 3.5\% of the between-person variance in mindwandering.

10. We stated that "the nature of people's activities... had almost no impact on the pleasantness of the topics their minds wandered to.” Evidence for this statement includes the facts that (a) 
although mind-wandering to an unpleasant topic was associated with less happiness $(p<$ .001), multilevel logistic regression revealed that the probability of mind-wandering to an unpleasant topic was unrelated to a person's activity ( $p>0.25)$, and (b) person-level regression revealed that differences in people's activities explained less than $1 \%$ of the between-person variance in the rate of mind-wandering to an unpleasant topic (Adj $\mathrm{R}^{2}=$ $0.0085, p<.05$ ) or a neutral topic (Adj $\mathrm{R}^{2}=0.0088, p<.01$ ), and less than $2 \%$ of the between-person variance in the rate of mind-wandering to a pleasant topic (Adj $\mathrm{R}^{2}=0.016, p$ $<.001)$

11. We stated: "time-lag analyses strongly suggested that mind-wandering in our sample was generally the cause-and not merely the consequence-of unhappiness." We used multilevel regression to determine whether there was a relationship between happiness in given sample ( $\mathrm{T})$ and mind-wandering in the previous sample $(\mathrm{T}-1)$ and/or the next sample $(\mathrm{T}+1)$. Table S1 presents the results of this analysis. The analysis yielded a significant negative relationship between mind-wandering at T-1 and happiness at time T (Model 1), but no significant relationship between mind-wandering at $\mathrm{T}+1$ and happiness at time $\mathrm{T}$ (Model 2). These findings were confirmed when mind-wandering at $\mathrm{T}-1$ and $\mathrm{T}+1$ were entered simultaneously into the regression (Model 3) and when mind-wandering at $\mathrm{T}$ was added as a control variable (Model 4). In short, across the four models we found a strong negative relationship between mind-wandering at $\mathrm{T}-1$ and happiness at $\mathrm{T}$, but no relationship between mind-wandering at T+1 and happiness at T. In other words, a person’s happiness was strongly related to whether they had been mind-wandering in the previous sample, but was unrelated to whether they were mind-wandering in the next sample. This is precisely what one would expect if mind-wandering caused unhappiness, and precisely the opposite of what 
one would expect if mind-wandering and unhappiness were related only because unhappiness causes mind-wandering. Although this does not preclude the possibility that unhappiness also caused mind-wandering, such an effect appears to play at most a modest role in the present results.

Table S1. Fixed effects estimates for Happiness at Time T

\begin{tabular}{|l|l|l|l|l|}
\hline & $\begin{array}{l}\text { Model 1 } \\
\mathrm{b}(\mathrm{SE})\end{array}$ & $\begin{array}{l}\text { Model 2 } \\
\mathrm{b}(\mathrm{SE})\end{array}$ & $\begin{array}{l}\text { Model 3 } \\
\mathrm{b}(\mathrm{SE})\end{array}$ & $\begin{array}{l}\text { Model 4 } \\
\mathrm{b}(\mathrm{SE})\end{array}$ \\
\hline $\begin{array}{l}\text { Mind-Wandering } \\
(\mathrm{T}-1)\end{array}$ & $-1.07(.331) * *$ & & $-1.21(.354) * *$ & $-1.34(.345) * * *$ \\
\hline $\begin{array}{l}\text { Mind-Wandering } \\
(\mathrm{T}+1)\end{array}$ & & $-0.365(.331)$ & $-0.294(.357)$ & $-0.511(.348)$ \\
\hline $\begin{array}{l}\text { Mind-Wandering } \\
(\mathrm{T})\end{array}$ & & & & $-9.04(.376) * * *$ \\
\hline
\end{tabular}

Note: $* * P<.01 ; \quad * * * P<.001$.

12. We stated: “The nature of people's activities explained $4.6 \%$ of the within-person variance in happiness and 3.2\% of the between-person variance in happiness, but mind-wandering explained $10.8 \%$ of within-person variance in happiness and $17.7 \%$ of between-person variance in happiness.” To compute the within-person variation, happiness scores were centered on each person's mean happiness. Then, OLS regression was used at the sample level to analyze the amount of within-person variance in happiness explained by mindwandering and by activity. To compute the between-person variation, person-level mean values of happiness, proportion of time spent in each mind-wandering state, and proportion of time spent in each activity were computed. Then, OLS regression was used at the person 
level to analyze the amount of between-person variance in happiness explained by mindwandering and by activity.

13. We stated: "The variance explained by mind-wandering was largely independent of the variance explained by the nature of activities, suggesting that the two are independent influences on happiness." While activity explained 3.2\% and mind-wandering explained $17.7 \%$ of between-person variance in happiness, together they explained $19.9 \%$ of betweenperson variance. While activity explained $4.6 \%$ and mind-wandering explained $10.8 \%$ of within-person variance in happiness, together they explained $14.8 \%$ of between-person variance. In each case, the variance explained by activity and mind-wandering together approached the sum of the variances that each factor explained on its own.

\section{SUPPORTING REFERENCES}

S1. D. Kahneman, A. B. Krueger, D. A. Schkade, N. Schwarz, A. A. Stone, Science 306, 1776 (2004).

S2. D. Kahneman, in Well-being: The foundations of hedonic psychology., D. Kahneman, E. Diener, N. Schwarz, Eds. (Russell Sage Foundation, New York, 1999), pp. 3-25.

S3. B. L. Fredrickson, D. Kahneman, J. Pers. Soc. Psychol. 65, 45 (1993).

S4. N. Schwarz, F. Strack, in Well-being: The foundations of hedonic psychology., D. Kahneman, E. Diener, N. Schwarz, Eds. (Russell Sage Foundation, New York, 1999), pp. 61-84.

S5. A. Krueger, D. Schkade, Journal of Public Economics 92, 1833 (2008). 